10. Unverferth D, Magorien R, Altschuld R, Kolibush A, Lewis $\mathrm{R}$, Leier $\mathrm{C}$ : 'The hemodynamic and metabolic advantages gained by a three-day infusion of dobutamine in patients with congestive cardiomyopathy. AM HEART J 1983;106:29.

11. Liang C, Sherman L, Doherty J, Wellington K, Lee V, Hood W: Sustained improvement of cardiac function in patients with congestive heart failure after short term infusions of dobutamine. Circulation 1984;69:113.

12. Leier CV, Huss P, Lewis RP, Unverferth DV: Drug-induced conditioning in congestive heart failure. Circulation 1982; 65:1382.

13. Applefeld MA, Newman KA, Grove WR, Sutton FJ, Roffman DS, Reed WP, Linberg SE: Intermittent, continuous outpatient dobutamine infusion in the management of congestive heart failure. Am .J Cardiol 1983;51:455.

14, LeJemetel TH, Keung E, Ribner HS, Davis R, Wexler J, Blaufox MD, Sonnenblick EH: Sustained beneficial effects of oral amrinone on cardiac and renal function in patients with severe heart failure. Am J Cardiol 1980;45:123.

15. Awan NA, Evenson MK, Needham KE, Evans TO, Hermano- vich J, Taylor CR, Amsterdam E, Mason DT: Hemodynamic effects of oral pirbuterol in chronic severe :ongestive heart failure. Circulation 1981;63:96

16. Baim DS, McDowell AV, Cherniles J, Monrad ES, Parker JA Edelson J, Braunwald E, Grossman W: Evaluation of a new bipyridine inotropic agent-milrinone-..in patients with severe congestive heart failure. N Engl J Med 1983;309:748.

17. Maskin CS, Forman R, Sonnenblick BH, Frishman WH LeJemtel TH: Failure of dobutamine to increase exercise capacity despite hemodynamic improvement in severe chronic heart failure. Am J Cardiol 1983;51:177.

18. Weber KT, Andrews V, Janicki JS: Cardiotonic agents in the management of chronic cardiac failure. $A$, Hiak' of 1982 103:639.

19. Kirlin PC, Pitt B: Hemodynamic effects of intravenous: prenalterol in severe heart failure. Am rardiol 1981 47:670.

20. LeJemtel TH, Sonnenblick EH: Should the failing heart be stimulated? N Engl J Med 1984;310:1;884

\title{
The hemodynamic determinants of the isovolumic index
}

\begin{abstract}
The isovolumic index is a recently described echocardiographic parameter of left ventricular function that is calculated as the ratio between the sum of the time of isovolumic contraction and relaxation divided by the ejection time. Although the individual components of this index may be altered by heart rate and loading conditions, an analysis of the net effect of such alterations on the isovolumic index has not been undertaken. Thus, dogs were instrumented with high-fidelity micromanometers in the left ventricle, ascending aorta, and left atrium to allow determination of the individual components of the isovolumic index and calculation of the index itself. Four sets of experiments were undertaken in random order. Left atrial pacing was used to increase heart rate by approximately $10 \mathrm{bpm}$ in five steps. Preload was elevated in five stages by saline infusions which caused successive increases of 1 to $2 \mathrm{~mm} \mathrm{Hg}$ in the left ventricular end-diastolic pressure. Systolic blood pressure was lowered or raised by approximately $10 \mathrm{~mm} \mathrm{Hg}$ per stage by three progressive, steady-state infusions of nitroprusside and phenylephrine, respectively. These experiments demonstrated little change in the isovolumic index over a broad range of heart rate. Increased left ventricular end-diastolic pressure and decreased systemic pressure caused shortening of the index. Multiple regression analysis of all experiments yielded the following: isovolumic index $=0.41-0.015$ (left ventricular end-diastolic pressure) +0.004 (systolic blood pressure); $r=0.57$, standard error $=0.13, p<0.0001$. Therefore, this investigation establishes the hemodynamic determinants of the isovolumic index and provides the basis for interpretation of directional changes in response to cardiac diseases and cardioactive drugs that can alter loading conditions. (AM HEART J 1986;112:791.)
\end{abstract}

\section{G. B. John Mancini, M.D., Harold Z. Friedman, M.D., John E. Hramiec, B.A., and Scott F. DeBoe, B.S. Ann Arbor, Mich.}

From Veterans Administration Medical Center, University of Michigan Medical School.

This study was supported by the Michigan Heart Association, Lathrup Village, Michigan; and by the Veterans Administration, Washington, I).C.

Received for publication Oct. 15, 1985; revision received Jan. 13, 1986: accepted Feb. 20, 1986.

Reprint requests: $Q$, B. John Mancini, M.D. Cardiology Section, VA Medical Center, 2215 Fuller Rd., Ann Arbor, MI 48105.
The isovolumic index is a recently described echocardiographic parameter of left ventricular function. ${ }^{1}$ It is an estimate of the sum of the total time of isovolumic contraction and relaxation divided by the ejection time and can be conveniently calculated from an $\mathrm{M}$-mode echocardiogram. The rationale for studying this index stems from two lines of reasoning. First, it has long been appreciated that in the 
setting of left ventricular failure, the preejection period lengthens while the ejection time diminishes. ${ }^{2-4}$ Second, it has become increasingly apparent that parameters of isovolumic relaxation are also abnormal under these circumstances. ${ }^{5-14}$ Thus, it was postulated that an "isovolumic index," as defined above, might be abnormal in appropriate patient subgroups and might be more sensitive in detecting these patients noninvasively than the traditional systolic time index (ratio of preejection time to ejection time) which ignores abnormalities of isovolumic relaxation.

Initial patient studies, ${ }^{1}$ demonstrated that the isovolumic index was abnormal in those with congestive cardiomyopathy and also in a substantial proportion with stable coronary artery disease. Despite these encouraging results, it was recognized that components of the index may be affected by heart rate and loading conditions. ${ }^{1,8,15-18}$ These possible effects require study before the potential clinical significance of directional changes in the isovolumic index secondary to ischemia ${ }^{19}$ or cardioactive drugs can be determined. Thus, the purpose of this investigation was to assess potential changes in the isovolumic index secondary to alterations of heart rate, preload, and afterload.

\section{METHODS}

Seventeen mongrel dogs of either sex $(25.2 \pm 4.2 \mathrm{~kg})$ were anesthetized with $35 \mathrm{mg} / \mathrm{kg}$ of sodium pentobarbital, intubated, and ventilated with a Harvard respirator. A left thoracotomy and left carotid arteriotomy were performed. The heart was supported in a preicardial cradle and high-fidelity micromanometers (No. 5F, Millar Instruments, Inc, Houston Texas) were inserted via a left carotid arteriotomy into the ascending aorta, via a left ventricular apical stab wound into the left ventricle, and via a left atriotomy into the left atrium and then through the mitral valve into the left ventricle. The catheters were made equisensitive by matching the systolic blood pressure readings of the left ventricular and the aortic manometers and by matching the high-gain left ventricular diastolic pressures of the left atrial and left ventricular catheters. Once achieved, the left atrial catheter was withdrawn through the mitral valve into the left atrium. Frequent checks for zero drift were made throughout the experiments. Left atrial pacing wires were sutured in place and wcre attached to an AV-120 Bloom stimulator (Bloom and Associates, Reading, $\mathrm{Pa}$.). A pair of subendocardial ultrasonic crystals were placed in the distribution of the left anterior descending coronary artery in the mid-equatorial plane of the ventricle. These were attached to a sonomicrometer 120 (Triton Technology, San Diego, Calif.), which was interfaced to a Tektronix 2213A oscilloscope (Tektronix, Inc., Beaverton, Or.). Proper crystal alignment was confirmed by oscilloscopic display of the crystal signals and by direct inspection at the conclusion of the experiment. All signals were recorded on an eight-channel Gould 2800S recorder (Gould Recording Systems, Cleveland, Ohio), and reported values are averages from four to six beats recorded at $200 \mathrm{~mm} / \mathrm{sec}$ paper speed during steady states.

Continuous recordings of left ventricular pressure, rate of change of pressure $(\mathrm{dP} / \mathrm{dt})$, aortic and atrial pressure, segment lengths, and ECG were made (Fig. 1). Left ventricular end-diastolic pressures were measured at the time when $\mathrm{dP} / \mathrm{dt}$ became positive, and this was considered the onset of isovolumic contraction. End systole was taken at peak negative $\mathrm{dP} / \mathrm{dt}$, and this was considered the beginning of isovolumic relaxation. ${ }^{20}$

The isovolumic contraction time was determined as the time from cnd-diastolic pressure to pcak positive $\mathrm{dP} / \mathrm{dt}^{21}$ (Fig. 2). The ejection time was determined as the time from peak positive to peak negative $\mathrm{dP} / \mathrm{dt}^{20,21}$ The isovolumic relaxation time was taken from peak negative $\mathrm{dP} / \mathrm{dt}$ to the point where left atrial and left ventricular diastolic pressures crossed over. ${ }^{22.26}$ The isovolumic index was defined as (IVC + IVR)/ET, where IVC = isovolumic contraction time, IVR $=$ isovolumic relaxation time, and ET = left ventricular ejection time. The systolic time index was defined as IVC/ET. Segment shortening was defined as segment excursion between end diastole and end systole divided by end-diastolic length and multiplied by 100 . To determine directional changes in relaxation, an estimate of the isovolumic relaxation time constant was determined by dividing the left ventricular pressure at peak negative $\mathrm{dP} / \mathrm{dt}$ by peak negative $\mathrm{dP} / \mathrm{dt}^{27.30}$

The experimental protocol was randomized into four phases with ample time between each to reattain steadystate hemodynamics. The effects of changing heart rate was assessed by incremental atrial pacing in steps of approximately $10 \mathrm{bpm}$ at five successive levels. The effects of volume loading were assessed by infusing saline in 50 to $150 \mathrm{cc}$ amounts to obtain five increasing levels of left ventricular end-diastolic pressure. Afterload was altered by three incremental infusions of nitroprusside and phenylephrine in order to cause steady-state reductions and elevations, respectively, of systolic pressure of approximately 10 to $15 \mathrm{~mm} \mathrm{Hg}$ per stage.

All results were analyzed by repeated measures analysis of variance. When overall significance was detected, a Bonferonni test was utilized to determine which stages were significantly different from control. ${ }^{31}$ A $p$ value of less than 0.05 was considered significant. Results are reported as mean \pm standard deviation. Data from all four phases were combined for a stepwise regression procedure to determine which combination of parameters best explained the variations in the isovolumic index. When hemodynamic instability, arrhythmias, non-steadystate conditions, crystal misalignment, or excessive zerodrift of pressure measurements were found, the data from all stages of that experiment were excluded from analysis. Full complements of data at each desired level were obtained in 12 dogs for the atrial pacing protocol, in eight dogs during saline loading, in 13 dogs during nitroprusside infusion, and in 11 dogs during phenylephrine infusion. 


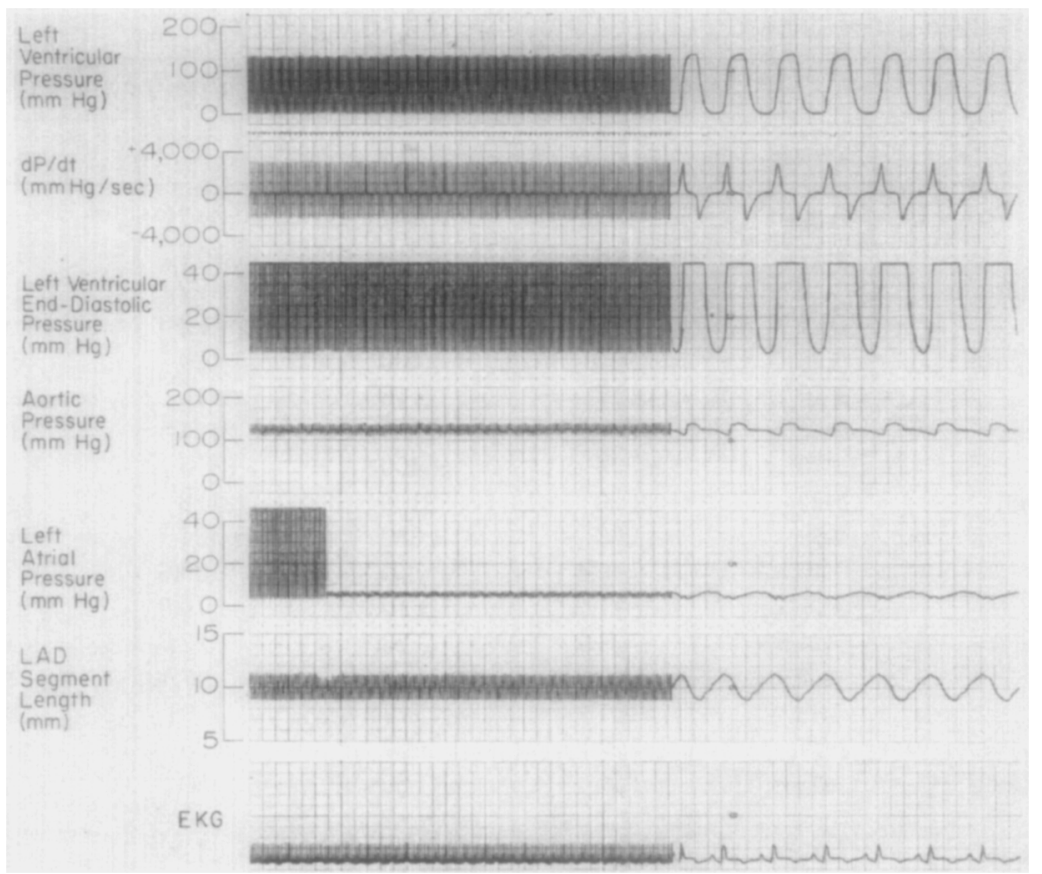

Fig. 1. Recordings at $2 \mathrm{~mm} / \mathrm{sec}$ and at $50 \mathrm{~mm} / \mathrm{sec}$ paper speed showing pressure, segment length, and electrocardiographic data. The fifth channel shows pressures as the left atrial manometer is pulled into the left atrium from the left ventricle. $\mathrm{dP} / \mathrm{dt}$ is the rate of change of left ventricular pressire with time.

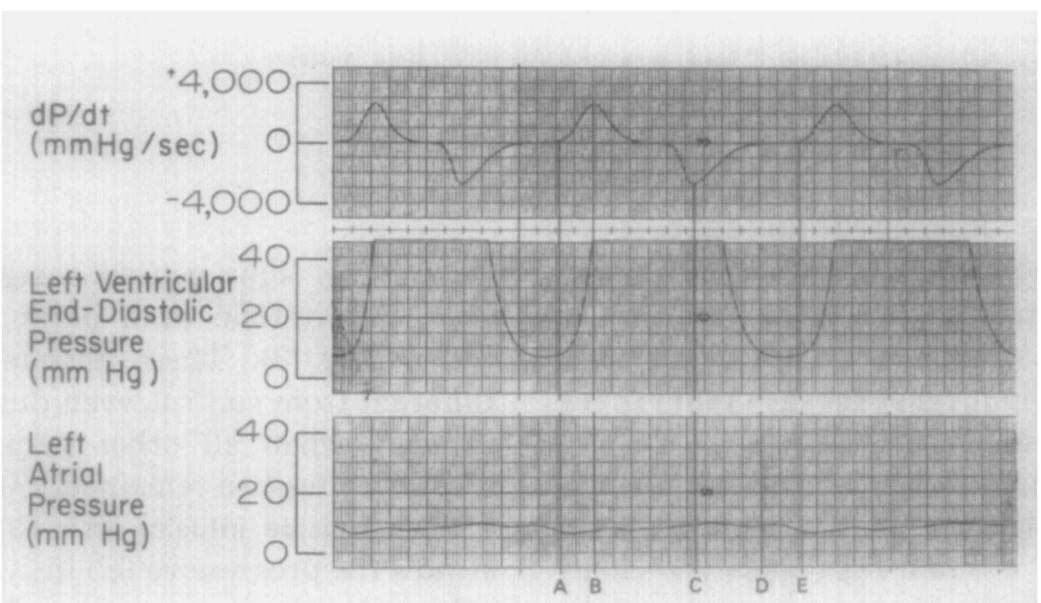

Fig. 2. Markers used for calculating time intervals are shown. $A B=$ isovolumic contraction time; $B C=$ ejection time; $C D=$ isovolumic relaxation time; $A E=$ full cycle length. Point $D$ is determined from the crossover of left ventricular diastolic pressure and left atrial pressure. $\mathrm{dP} / \mathrm{dt}$ is the rate of change of left ventricular pressure with time.

\section{RESULTS}

Atrial pacing $(n=12)$. Table I summarizes the results of the atrial pacing protocol. Stepwise increments in heart rate occurred from control values of $129 \pm 17$ to $177 \pm 17 \mathrm{bpm}$. Left ventricular enddiastolic pressure and systolic blood pressure did not change. Peak negative $\mathrm{dP} / \mathrm{dt}$ and the isovolumic relaxation time constant also showed no significant changes. A rate-dependent increase in peak positive
$\mathrm{dP} / \mathrm{dt}$ and deterioration in segment shortening were noted at the highest levels of pacing. All time intervals progressively shortened with increasing heart rate, and this was greatest for the ejection time and least for the isovolumic contraction time. This resulted in a small but significant increase in the systolic time index (IVC/ET). Although a downward trend in the isovolumic index was noted, these changes were not significant. Excluding the last two 
Table I. Effects of incremental atrial pacing on hemodynamics and time intervals

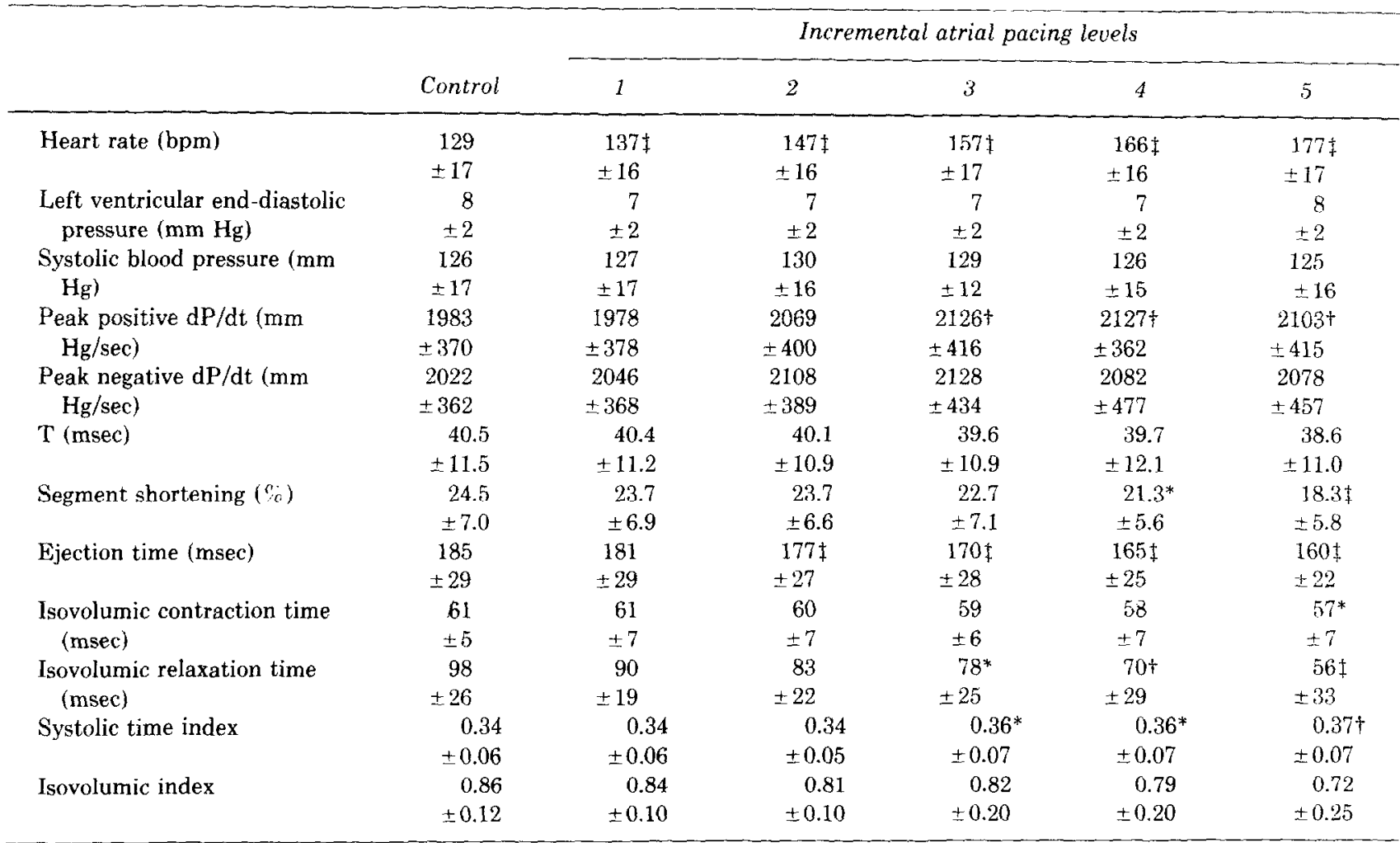

Values are mean \pm S.D.

$\mathrm{dP} / \mathrm{dt}=$ rate of pressure change with respect to time, $\mathrm{T}$ is the isovolumic relaxation time constant.

${ }^{*} p<0.05$ vs control.

$\dagger p<0.01$ vs control.

$\ddagger p<0.001$ vs control.

rates of extreme tachycardia when fractional shortening was mildly impaired, the isovolumic index showed no significant change over a heart rate range of approximately $30 \mathrm{bpm}$, whereas the systolic time index showed a very mild prolongation.

Saline loading $(n=8)$. Left ventricular end-diastolic pressure changed significantly in a stepwise fashion from $8 \pm 2$ to $16 \pm 2 \mathrm{~mm} \mathrm{Hg}$ (Table II). Heart rate showed no significant change, whereas systolic blood pressure rose significantly with the highest infusions. The elevation during stage four, however, did not reach statistical significance. Peak positive $\mathrm{dP} / \mathrm{dt}$ was elevated relative to control during the last three infusions. Peak negative $\mathrm{dP} / \mathrm{dt}$ remained constant, whereas the isovolumic relaxation time constant increased from $40 \pm 13 \mathrm{msec}$ to $45 \pm 11$ and $46 \pm 12 \mathrm{msec}$ during the fourth and fifth stages, respectively. Segment shortening showed the expected improvement with volume loading. The ejection time showed progressive prolongation in the face of progressive shortening of the isovolumic contraction time and a constant isovolumic relax- ation time. This caused a significant decrease in both the systolic time index and the isovolumic index (Fig. 3). These changes were significantly different from control, even during the first stage of infusion when all other parameters except enddiastolic pressure remained unchanged.

Nitroprusside infusion $(n=13)$. Table III demonstrates the progressive fall in systolic blood pressure achieved with nitroprusside infusion. This was associated with a 10 to $12 \mathrm{bpm}$ increase in heart rate and a 2 to $3 \mathrm{~mm} \mathrm{Hg}$ fall in left ventricular end-diastolic pressure. Both peak positive and negative $\mathrm{dP} / \mathrm{dt}$ fell during this experiment, whereas segment shortening remained constant and the isovolumic relaxation time constant shortened. Significant abbreviation of both isovolumic contraction and relaxation times in conjunction with a constant ejection time resulted in a significant decrease in both the isovolumic index and the systolic time index (Fig. 4). It should be noted that changes of heart rate in this experiment cannot account for the changes in the isovolumic index or the systolic time index (see Table I). 
Table II. The effects of volume loading on hemodynamics and time intervals

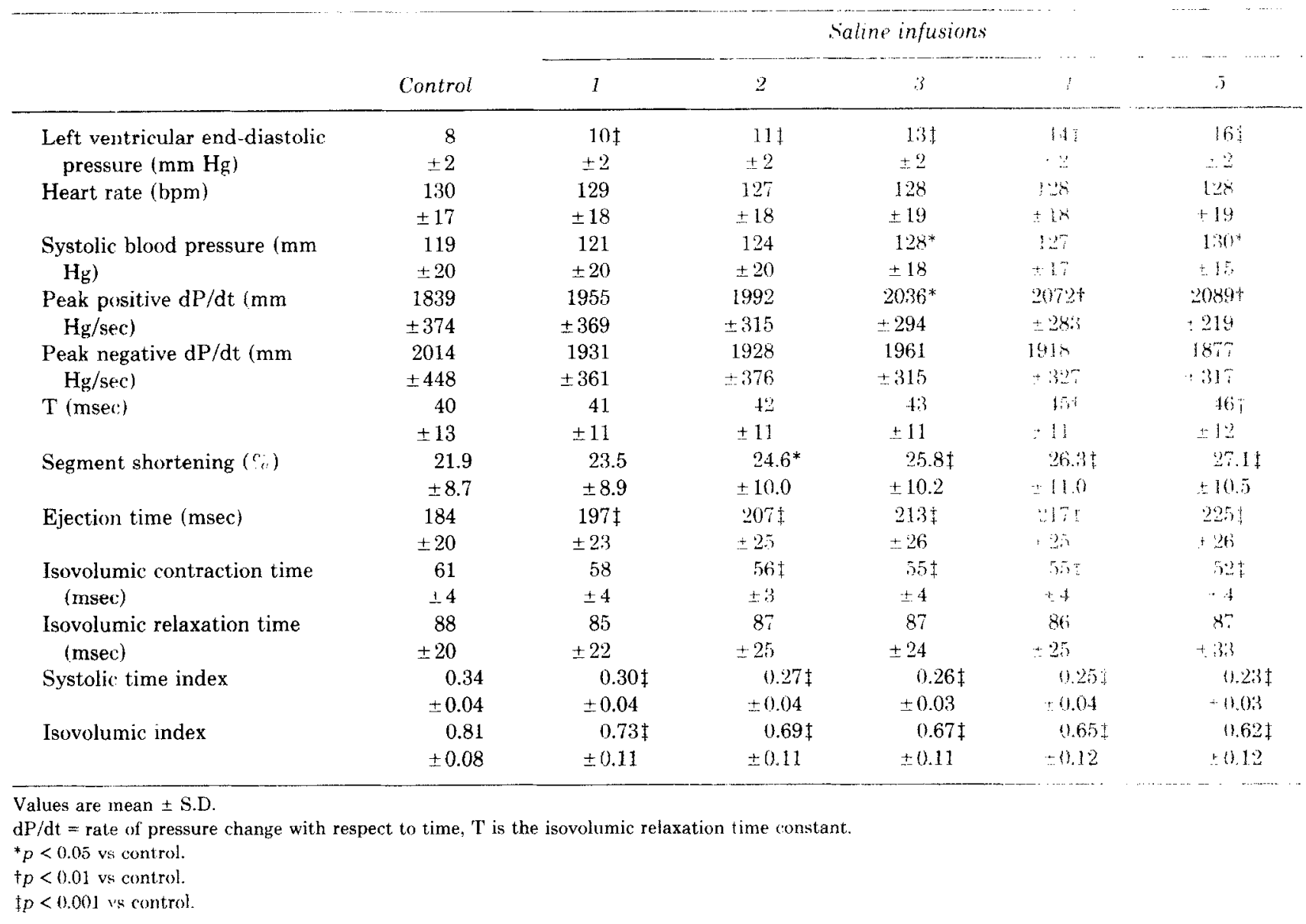

Similarly, the decrease in left ventricular enddiastolic pressure would not be expected to cause a fall in these indices (see Table II).

Phenylephrine infusion $(n=11)$. Systolic blood pressure rose significantly by approximately $15 \mathrm{~mm}$ $\mathrm{Hg}$ in each stage (Table IV, Fig. 4). A slight but significant fall in heart rate of approximately $7 \mathrm{bpm}$ occurred in the final stage, and the left ventricular end-diastolic pressure rose during the final two stages of the protocol. Peak positive and negative $\mathrm{dP} / \mathrm{dt}$ showed small increases. Fractional shortening remained constant and the isovolumic relaxation time constant showed the anticipated prolongation, which was paralleled by a significant lengthening of the isovolumic relaxation time. Isovolumic contraction time remained constant and the ejection time showed mildly progressive prolongation that was not significant until stage three. Compared to saline loading, the increase in left ventricular end-diastolic pressure with phenylephrine infusion was not manifested in a progressive shortening of either the isovolumic index or the systolic time index in the face of rising systemic pressure. Both indices remained constant.

Regression analysis. Stepwise regression analysis on all data yielded: IVI $=0.41-0.015$ LVEDP $+0.004 \mathrm{SBP}, r=0.57$, standard error $=0.13$; where IVI is the isovolumic index, LVEDP is the leftventricular end-diastolic pressure, and SBP is the systolic blood pressure. All factors were highly significant at $p<0.0001$. Heart rate effects were not significant in this analysis. In contrast, regression of the systolic time index (STI) showed: STI = $0.12+0.009$ HR -0.007 LVEDP +0.001 SBP, $r=0.63, \mathrm{SEE}=0.05$; where $\mathrm{HR}$ is heart rate. Thus, the STI was slightly more correlated overall with the hemodynamic and heart rate effects.

\section{DISCUSSION}

The isovolumic index is determined by a complex interplay between three distinct phases of the cardiac cycle: ejection time, isovolumic contraction time, and isovolumic relaxation time. Therefore, the conclusions of this investigation depend on the validity 


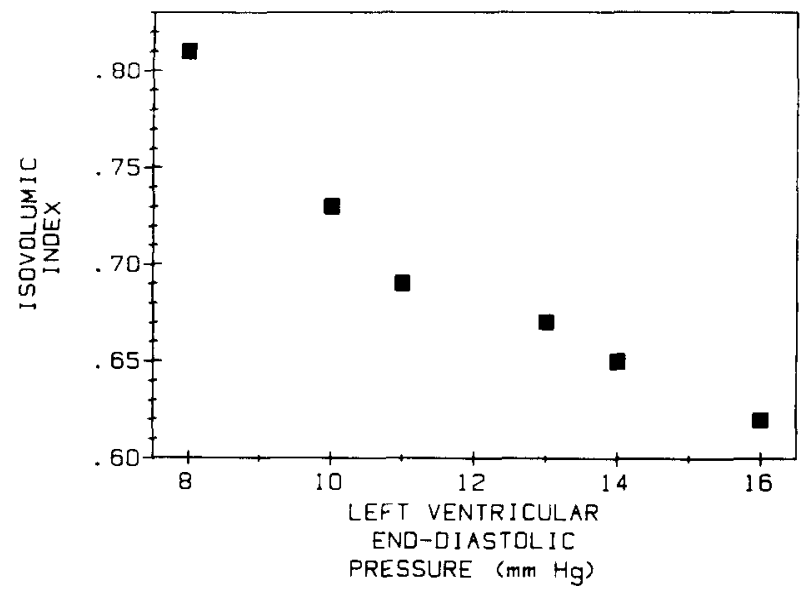

Fig. 3. The effects of saline loading on the left ventricular end-diastolic pressure and the isovolumic index.

of the observed directional changes of these intervals. The ejection time has previously been shown to vary inversely with heart rate, ${ }^{2,15}$ and to increase with enhanced left ventricular filling,,32 and enhanced systemic afterload. ${ }^{33}$ Decreased afterload prolongs the left ventricular ejection time if stroke volume is augmented. ${ }^{34}$ This study confirmed these findings, except that the ejection time remained constant during nitroprusside infusion. This may have resulted from a lack of increase in stroke volume because of concomitant lowering of filling pressures. This is corroborated by the failure of myocardial shortening to increase (Table IV).

The isovolumic contraction time revealed only a slight fall with increasing heart rate changes and a decrease during both saline loading and nitroprusside infusion. Again, these findings are in keeping with prior observations. ${ }^{15,16}$ During phenylephrine infusion, however, this interval did not change, in contrast to the work of Harris et al.$^{35}$ and Shaver et al. ${ }^{33}$ that demonstrated prolongation with pressor agents. In the current study, preservation of myocardial shortening by maintenance and enhancement of left ventricular filling may have prevented changes in the stroke volume and, hence, isovolumic contraction time is expected to remain constant (Table III). ${ }^{36}$

The third important interval is the isovolumic relaxation time. This interval has been shown to be affected by the level of aortic closing pressure, the left atrial pressure, and the rate of left ventricular pressure fall. ${ }^{14,17,37}$ In this study, no significant alteration in isovolumic relaxation time was noted during saline infusion despite significant elevations of left ventricular end-diastolic pressures and left atrial

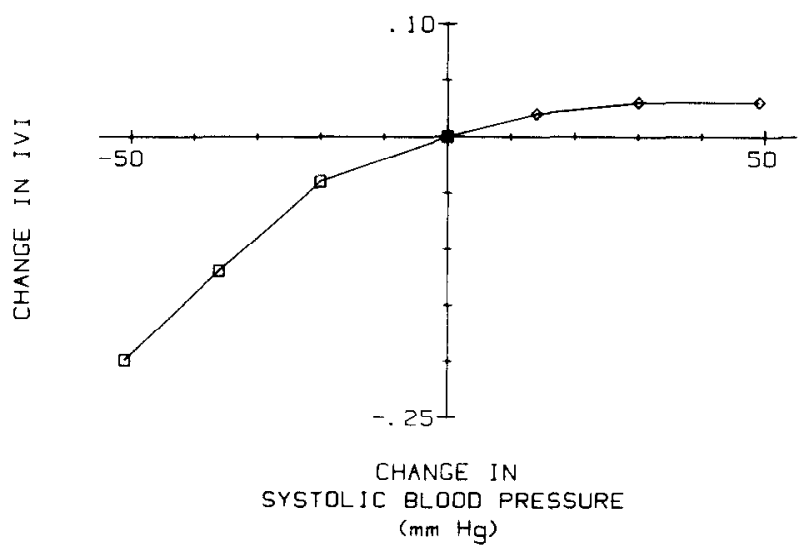

Fig. 4. The change in the isovolumic index (IVI) is shown as a function of the change in systolic blood pressure. Squares represent mean observations during nitroprusside infusions and diamonds represent those during phenylephrine infusion.

pressures. This can be explained by the offsetting effects of elevated systolic pressure and the prolonged rate of isovolumic relaxation. ${ }^{14,17,37-39}$ This was apparent during the phenylephrine infusions, when more marked elevations in systolic pressure were associated with prolongation of the isovolumic relaxation time constant and the isovolumic relaxation time despite an increase in left ventricular enddiastolic pressures. Moreover, the opposite effects were noted during nitroprusside infusion, i.e., isovolumic relaxation time shortened in parallel with a shorter isovolumic relaxation time constant and decreasing systemic pressures. These latter effects offset the mild fall of left ventricular end-diastolic pressure that might have prolonged the isovolumic relaxation period. The effect of heart rate on the isovolumic relaxation time interval is less clearly defined, and investigators have reported no change, ${ }^{18,37,40,41}$ increases, ${ }^{14}$ or decreases..$^{13}$ This inter$\mathrm{val}$ is partially determined by the rate of fall of left ventricular pressure and the isovolumic relaxation time constant, both of which have been shown to be more rapid with increasing heart rate. ${ }^{38,42}$ In the current investigation, the isovolumic relaxation time was not significantly different with changes in rate of approximately $20 \mathrm{bpm}$, but at higher rates a progressive decrease appeared. These findings cannot be explained by changes in systemic pressures or filling pressures, because these remained constant. In addition, peak negative $\mathrm{dP} / \mathrm{dt}$ and the isovolumic relaxation time constant were not significantly changed, although the latter tended toward progressive shortening. The use of the pressure crossover point of left ventricular and left atrial pressures may have been less closely related to mitral valve opening 
Table III. The effects of progressive hypotension induced by nitroprusside on hemodynamics and time intervals

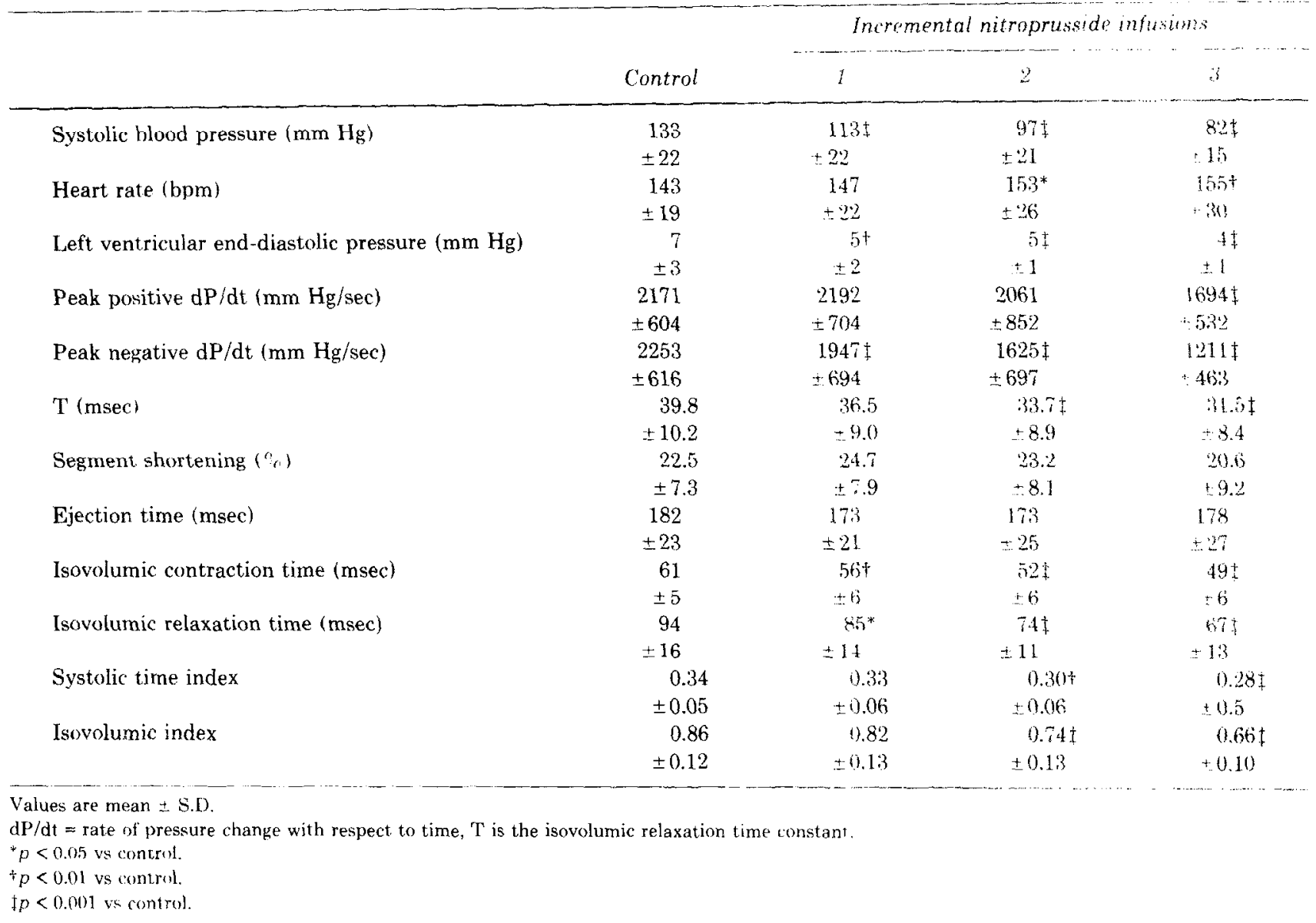

during rapid left atrial pacing than at lower rates. ${ }^{23}$ Left atrial pacing caused a very rapid and marked pressure rise in the left atrium. It is conceivable that the rapid pressure rise in the left atrium caused a progressive underestimation of actual mitral valve opening and the end of the isovolumic relaxation period. Thus, the effects of very large increases in heart rate in this study must be considered tentative, whereas observations made within rate changes of approximately $20 \mathrm{bpm}$ above control are in keeping with no significant alteration of either the isovolumic relaxation time or the isovolumic index. The results of multiple regression analysis confirm that heart rate changes do not affect the isovolumic index.

Limited observations in dogs reported in the initial descriptive study of the isovolumic index showed an inverse relation between fractional shortening and the isovolumic index. ${ }^{1}$ Fractional shortening was altered by increasing left ventricular enddiastolic pressures in that study. The results shown in Table II confirm those results and also confirm the speculated preload dependence of the isovolum- ic index. Moreover, this dependence can be explained by prolongation of the ejection time and shortening of the isovolumic contraction time by saline loading. Although no overall correlation between the isovolumic index and ejection fraction was noted in patients, it is not yet clear whether directional changes in the isovolumic index will show a relationship to changes in shortening in the absence of loading changes; this will require further elucidation. Determination of the isovolumic index in patients at rest failed to reveal any relationship between this index and heart rate or systemic blood pressure. ${ }^{1}$ The current findings confirm the lack of major heart rate effects on the isovolumic index. In contrast, systemic pressure changes did influence the measurement of the isovolumic index, particularly with nitroprusside infusion. This drug brought about a shortening of both isovolumic periods and maintenance of a stable ejection time. Thus, the isovolumic index shortened.

Although the isovolumic index is less correlated overall with hemodynamic and heart rate changes, a comparison of the directional changes in the isovo- 
Table IV. The effects of progressive infusions of phenylephrine on hemodynamics and time intervals

\begin{tabular}{|c|c|c|c|c|}
\hline & \multirow[b]{2}{*}{ Control } & \multicolumn{3}{|c|}{$\begin{array}{c}\text { Incremental } \\
\text { phenylephrine infusions }\end{array}$} \\
\hline & & 1 & 2 & 3 \\
\hline \multirow[t]{2}{*}{ Systolic blood pressure ( $\mathrm{mm} \mathrm{Hg}$ ) } & 130 & $144 \dagger$ & $160 \ddagger$ & $179 \ddagger$ \\
\hline & \pm 15 & \pm 13 & \pm 16 & \pm 18 \\
\hline \multirow[t]{2}{*}{ Heart rate $(\mathrm{bpm})$} & 147 & 146 & 143 & $140 \ddagger$ \\
\hline & \pm 15 & \pm 14 & \pm 13 & \pm 13 \\
\hline \multirow[t]{2}{*}{ Left ventricular end-diastolic pressure $(\mathrm{mm} \mathrm{Hg}$ ) } & 7 & 8 & $11 \dagger$ & $15 \ddagger$ \\
\hline & \pm 2 & \pm 3 & \pm 6 & \pm 8 \\
\hline \multirow[t]{2}{*}{ Peak positive $\mathrm{dP} / \mathrm{dt}(\mathrm{mm} \mathrm{Hg} / \mathrm{sec})$} & 2082 & 2178 & 2191 & $2286 \dagger$ \\
\hline & \pm 467 & \pm 425 & \pm 309 & \pm 292 \\
\hline \multirow[t]{2}{*}{ Peak negative $\mathrm{dP} / \mathrm{dt}(\mathrm{mm} \mathrm{Hg} / \mathrm{sec})$} & 2168 & 2283 & $2343^{*}$ & 2313 \\
\hline & \pm 518 & \pm 520 & \pm 549 & \pm 547 \\
\hline \multirow[t]{2}{*}{$\mathrm{T}$ (msec) } & 41.2 & 45.1 & $50.4^{*}$ & $57.1 \ddagger$ \\
\hline & \pm 12.6 & \pm 14.2 & \pm 22.9 & +24.0 \\
\hline \multirow[t]{2}{*}{ Segment shortening $(\%)$} & 22.9 & 22.5 & 23.4 & 21.4 \\
\hline & \pm 5.7 & \pm 6.4 & \pm 7.9 & \pm 10.3 \\
\hline \multirow[t]{2}{*}{ Ejection time (msec) } & 184 & 187 & 191 & $196 \dagger$ \\
\hline & \pm 23 & \pm 24 & \pm 22 & \pm 18 \\
\hline \multirow[t]{2}{*}{ Isovolumic contraction } & 62 & 63 & 62 & 63 \\
\hline & \pm 8 & \pm 7 & \pm 6 & \pm 6 \\
\hline \multirow[t]{2}{*}{ Isovolumic relaxation time (msec) } & 87 & 93 & $98 \dot{\dagger}$ & $103 \ddagger$ \\
\hline & \pm 17 & \pm 20 & \pm 27 & \pm 27 \\
\hline \multirow[t]{2}{*}{ Systolic time index } & 0.34 & 0.34 & 0.33 & 0.32 \\
\hline & \pm 0.04 & \pm 0.04 & \pm 0.04 & \pm 0.03 \\
\hline \multirow[t]{2}{*}{ Isovolumic index (msec) } & 0.82 & 0.84 & 0.85 & 0.85 \\
\hline & \pm 0.12 & \pm 0.14 & \pm 0.15 & \pm 0.15 \\
\hline
\end{tabular}

Values are mean \pm S.D.

$\mathrm{dP} / \mathrm{dt}=$ rate of pressure change with respect to time; $\mathrm{T}$ is the isovolumic relaxation time constant.

$* p<0.05$ us control

$\dagger p<0.01$ vs control.

$\ddagger p<0.001$ vs control.

lumic index and the systolic time index (isovolumic contraction divided by the ejection time) shows that both indexes respond in a very similar fashion to load changes. However, it is clear that the ultimate advantage of the isovolumic index might be in those situations demonstrating selective abnormalities of isovolumic relaxation or when subtle abnormalities of both isovolumic contraction and relaxation are present. Indeed, the isovolumic index has been found to be abnormal in a small group of coronary patients with normal ratios of preejection time to ejection time, ${ }^{1}$ underscoring a potential superiority of the isovolumic index despite similar load dependency.

In conclusion, this study establishes the hemodynamic determinants of the isovolumic index. It has been shown that the index is a load-dependent parameter that is independent of heart rate changes. The index shows a fall with decreasing systemic pressure and with physiologic increases in left ventricular end-diastolic pressure. Minimal change was noted in response to rising systemic pressure. A potential deterioration (prolongation), however, may have been offset by the progressively increased left ventricular end-diastolic pressure during phenylephrine infusion. As with other load-dependent parameters such as the ejection fraction or isovolumic parameters, detailed knowledge of the hemodynamic determinants of the isovolumic index are required before the significance of its directional changes in response to cardioactive drugs or ischemia can be determined. Thus, this investigation provides the framework for future applications of the isovolumic index in the clinical setting.

The authors wish to acknowledge the secretarial assistance of Diane Bauer and Marjorie LeRoy.

\section{REFERENCES}

1. Mancini GBJ, Costello D, Bhargava V, Lew W, LeWinter M, Karliner JS: The isovolumic index: A new noninvasive approach to the assessment of left ventricular function in man. Am J Cardiol 1982;50:1401.

2. Weissler AM, Harris WS, Schoenfeld CD: Systolic time intervals in heart failure in man. Circulation 1968;37:149.

3. Weissler AM, Harris WS, Schoenfeld CD: Bedside lechniques 
for the evaluation of ventricular function in man. Am $J$ Cardiol 1969;23:577.

4. McDonald IG, Hobson ER: A comparison of the relative value of noninvasive techniques--echocardiography, systolic time intervals and apexcardiography -in the diagnosis of primary myocardial disease. AM HEART J 1974;88:454.

5. Grossman W, McLaurin LP: Diastolic properties of the left ventricle. Ann Intern Med 1976;84:316.

6. Grossman W, Barry WH: Diastolic pressure-volume relations in the diseased heart. Fed Proc 1980;39:148.

7. Gibson DG, Prewitt TA, Brown DJ: Analysis of left ventricular wall movement during isovolumic relaxation and its relation to coronary artery disease. Br Heart J 1976; 38:1010.

8. Lewis BS, Gotsman MS: Current concepts of left ventricular relaxation and compliance. AM HEART J 1980;99:101.

9. Hiroto Y: A clinical study of left ventricular relaxation. Circulation 1980;62:756.

10. Rousseau MF, Veriter C, Detry JMR, Brasseur L, Pouleur H Impaired early left ventricular relaxation in coronary artery disease: Effects of intracoronary nifedipine. Circulation 1980:62:764.

11. Rubenstein JJ, Pohost GM, Foster JR: The echocardiographic determination of isovolumic relaxation period in patients with normal and diseased coronary arteries (abstr). Clin Res 1973;31:446.

12. D'Angelo $\mathrm{R}$, Shah $\mathrm{N}$, Rubler $\mathrm{S}$ : Diastolic time intervals in ischemic and hypertensive heart disease: A comparison of isovolumic relaxation time and rapid filling time with systolic time intervals. Chest 1975;68:56.

13. Benchimol A, Ellis JG: A study of the period of isovolumic relaxation in normal subjects and in patients with heart disease. Am J Cardiol 1967;19:196.

14. Lewis BS, Lewis N, Sapoznikov D, Gotsman MS: Isovolumic relaxation period in man. AM HEART J 1980;100:490.

15. Lewis RP, Rittgers SE, Forester WF, Boudoulas H: A critical review of the systolic time intervals. Circulation 1977; 56:146.

16. Hassan S, Turner P: Systolic time intervals: A review of the method in the non-invasive investigation of cardiac function in health, disease and clinical pharmacology. Postgrad Med J 1983;59:423.

17. Gamble WH, Shaver JH, Alvarez RF, Salerni R, Reddy PS: A critical appraisal of diastolic time intervals as a measure of relaxation in left ventricular hypertrophy. Circulation 1983;68:76.

18. Bahler RC, Vrobel TR, Martin P: The relation of heart rate and shortening fraction to echocardiographic indexes of left ventricular relaxation in normal subjects. J Am Coll Cardiol 1983;2:926.

19. Vadenbossche $J$-L, Taylor JE, Karliner $J$; Left ventricular functional recovery from exercise does not differ between normals and patients with coronary heart disease (abstr.) Circulation 1983;68(suppl III):III-10.

20. Abel FL: Maximal negative $\mathrm{dP} / \mathrm{dt}$ as an indicator of end of systole. Am J Physiol 1981:240:H676.

21. Mahler F, Ross J Jr, O'Rourke RA, Covell JW: Effects of changes in preload, afterload and inotropic state on ejection and isovolumic phase measures of contracticility in the conscious dog. Am J Cardiol 1975;35:626.

22. Rubenstein JJ, Pohost GM, Dinsmore RE, Harthorne JW: The echocardiographic determination of mitral valve opening and closure: Correlation and hemodynamic studies in man. Circulation 1975;51:98.

23. Tsakiris AG, Gordon DA, Padiyar R, Frechette D: Relation of mitral valve opening and closure to left atrial and ventricular pressures in the intact dog. Am J Physiol 1978;234:H146.
24. Laniado S, Yellin E, Kotler M, Levy L, Stadler J, Terdiman $R$ : A study of the dynamic relations between the mitral valve echogram and phasic mitral flow. Circulation 1975;51:104.

25. Pohost GM, Dinsmore RF, Rubenstein JJ, O'Keefe DD, Grantham RN, Scully HE, Beierholm EA, Frederickson JW, Weisfeldt ML, Daggett WH: The echocardiogram of the anterior leaflet of the mitral valve. Correlation with hemodynamic; and cineroentgenographic studies in dogs. Circulation 1975;51:88.

26. Laniado S, Yellin EL, Miller H, Frater RWM: Temporal relation of the first heart sound to closure of the mitral valve. Circulation 1973;47:1006.

27. Karliner JS, LeWinter MM, Mahler F. Engler R, O'Rourke RA: Pharmacologic and hemodynamic influences of the rate of isovolumic left ventricular relaxation in the normal conscious dog. J Clin Invest 1977;60:511.

28. Hirota Y: A clinical study of left veniricular relaxation. Circulation 1980;62:756.

29. Katayama K, Kumada T, Fujii T, Moritani K, Miura T, Toma Y, Kohno M, Yoshino F, Ogawa H, Ozaki M, Matsuzaki M, Matsuda Y, Kusukawa R: Clinical characteristics of left ventricular pressure decline during isovolumic relaxation in normal and diseased hearts. AM HEART I 1984;107:332.

30. Mirsky l: Assessment of diastolic function: Suggested meth. ods and future considerations. Circulation 1984;69:836.

31. Neter J, Wasserman W: Applied linear statistical models. Homewood, Ill., 1974, Richard D. Irwin, Inc. p 382.

32. Lewis RP, Boudoulos H, Welch TG. Forester WF: Usefulness of systolic time intervals in coronary artery disease. Am J Cardiol 1976;37:787.

33. Shaver JA, Kroetz FW, Leonard J., Paley HW: The effect of steady-state increases in systolic arterial pressure on the duration of left ventricular ejection tmo of Clin Invest 1968:47:217.

34. Sawayama $\mathrm{T}$, Ochiai $\mathrm{M}$, Marumato $\mathrm{S}$. Mastuura $\mathrm{T}$, Niki I: Influence of amyl nitrate inhalation on the systolic time intervals in normal subjects and in patients with ischemic heart disease. Circulation 1969;40:327.

35. Harris WS, Schoenfeld CD, Weissler AM: Effects of adrenergic receptors activation and blockade on the systolic preejection period, heart rate and arterial pressure in man. I Clin Invest 1967;46:1704

36. Matsuura T, Goodyer AVN: Effects of a pressure load on foft ventricular systolic time intervals. Am of Physios 1973; 224:80.

37. Weisfeldt ML, Scully HE, Frederiksen 3: Hemodynamic determinants of maximum negative $\mathrm{dP} / \mathrm{dt}$ and periods of diastole. Am d Physiol 1974:227:613.

38. Raff GL, Glantz SA: Volume loading slows left ventricular isovolumic relaxation rate: Evidence of load-dependent relaxation in the intact dog heart. Circ Res 1981;48:813.

39. Gaasch WH, Blaustein AS, Andrias CW, Donahue RP, Avitall B: Myocardial relaxation. II. Hemorlynamic determinants of rate of left ventricular isovolumic pressure decline. Am \& Physiol 1980;239:H1.

40. Cieslinski A, Jui WKK, Oldershaw PJ, Gregoratos $G$, Gibson D: Interaction between systolic and diastolic time intervals in atrial fibrillation. Br Heart $J$ 1984;51:4:31.

41. Wiggers CJ: Studies on the consecutive phases of the cardiac cycle. II. The laws governing the relative durations of ventric. ular systole and diastole. Am I Physiol 1921;56:439.

42. Fredericksen JW, Weiss JL, Weisfeldt ML: Time constant of isovolumic pressure fall: Determinant: in the working left ventricle. Am I Physiol 1978:235:H70! 